\title{
Muséologies
}

Les cahiers d'études supérieures

muséologies

\section{Back to the Future : art contemporain du Moyen-Orient et expositions temporaires au British Museum}

\section{Monia Abdallah}

Volume 9, numéro 1, 2018

Monde des Arts / Arts des mondes

URI : https://id.erudit.org/iderudit/1052627ar

DOI : https://doi.org/10.7202/1052627ar

Aller au sommaire du numéro

Éditeur(s)

Association Québécoise de Promotion des Recherches Étudiantes en

Muséologie (AQPREM)

ISSN

1718-5181 (imprimé)

1929-7815 (numérique)

Découvrir la revue

Citer cet article

Abdallah, M. (2018). Back to the Future : art contemporain du Moyen-Orient et expositions temporaires au British Museum. Muséologies, 9(1), 45-58.

https://doi.org/10.7202/1052627ar
Résumé de l'article

The confrontation between contemporary and ancient art, within the framework of temporary exhibitions or in the context of permanent collections, is not new, and examples are numerous. This article shows, through a description of a variety of temporary exhibitions organized by the British Museum, bringing together contemporary Middle Eastern and ancient Islamic art, the ideological consequences of such juxtapositions which consistently favour continuity over rift.
Tous droits réservés (C) Association Québécoise de Promotion des Recherches Étudiantes en Muséologie (AQPREM), 2018
Ce document est protégé par la loi sur le droit d'auteur. L'utilisation des services d'Érudit (y compris la reproduction) est assujettie à sa politique d'utilisation que vous pouvez consulter en ligne.

https://apropos.erudit.org/fr/usagers/politique-dutilisation/ 
Article deux

Back to the Future: art contemporain du Moyen-Orient et expositions temporaires au British Museum

Monia Abdallah 
Monia Abdallah est diplômée de l'École des hautes études en sciences sociales (EHESS, Paris). Actuellement professeure d'histoire de l'art à l'Université du Québec à Montréal, ses recherches portent sur les pratiques artistiques au Moyen-Orient et sur les liens entre Art, Islam et Modernité. Avant de rejoindre l'UQÀM, elle a effectué un postdoctorat à University of Toronto et a été boursière postdoctorale de la Andrew W Mellon Foundation au Courtauld Institute of Art (Londres) où elle a enseigné un cours sur l'art contemporain du Moyen-Orient. 
L'association ou la confrontation entre art contemporain et art ancien dans le cadre d'expositions temporaires ou dans le contexte de collections permanentes n'est pas récente et les exemples sont nombreux. Dans le contexte français, c'est dès 1947 que l'on choisit d'exposer des œuvres de Picasso dans la Grande Galerie du Louvre, musée qui commande en 1953 à Georges Braque l'œuvre Les Oiseaux pour le plafond de l'ancienne antichambre du roi. Les interventions d'artistes vivants dans les collections permanentes se multiplieront dès les années $1970^{1}$ et les confrontations entre art du présent et art du passé lors d'expositions temporaires se développeront à partir des années 1980². Au British Museum, c'est en 1994 qu'est organisée une des expositions temporaires marquantes réunissant art contemporain et art du passé. Intitulée Time machine. Ancient Egypt and Contemporary Art, cette exposition faisait dialoguer artefacts pharaoniques et art contemporain international ${ }^{3}$. Ces choix muséographiques et curatoriaux participent surtout de la fonction d'animation et de médiation des musées ${ }^{4}$ et, aujourd'hui, comme le souligne le conservateur français et actuel directeur du Musée national du Château de Versailles Laurent Salomé, «[...] les confrontations entre art contemporain et collections sont devenues hélas une forme banale d'animation culturelle ${ }^{5}$ ". Toutefois, dans ce qui suit, il s'agira de montrer au travers d'une description de différentes expositions temporaires, - la plupart ont eu lieu dans un espace qui leur est spécifiquement dédié dans la galerie John Addis - réunissant art contemporain du Moyen-Orient ${ }^{6}$ et art islamique historique ${ }^{7}$ organisées par le British Museum de 2004 à 2010, les conséquences idéologiques de telles confrontations lorsque celles-ci instrumentalisent systématiquement l'art contemporain d'une vaste région porteuse d'une pluralité de cultures pour n'en offrir qu'une vision homogénéisante, réductrice, confortant les préjugés et alimentant les rêves passéistes. Dans deux autres articles précédemment publiés, nous avons pu montrer que l'exposition d'œuvres contemporaines du Moyen-Orient dans la collection permanente d'art islamique historique au British Museum menait à la réactivation de la notion de civilisation islamique et faisait fi des périodes coloniale et décoloniale, et des ruptures qu'elles ont introduites. Nous avons aussi montré que ce choix muséographique, aujourd'hui adopté par de nombreuses autres institutions dans le monde, aboutissait à ne percevoir ces œuvres
1 Voir ALLEMAND-COSNEAU Claude, d'ANTHENAISE Claude, HUBER Thomas, SALOMÉ Laurent, de CHASSÉ Éric, « Les pratiques récentes de mixité entre art actuel et art ancien: le contemporain dans les musées ", PERSPECTIVE la revue de l'INHA, 2009-4, p. 496-509.

2 Voir ISUANI Yanina, "Des expositions de confrontation entre art du présent et art du passé ", entretien avec JeanHubert Martin, Marges. Revue d'art contemporain, $\mathrm{n}^{\circ} 8,2008$, p. 110-118.

3 Cette exposition ne fait pas partie du corpus ici étudié dans la mesure où elle n'a aucun lien avec l'Islam, n'impliquant aucun objet de l'art islamique historique, n'ayant aucun lien avec la galerie John Addis du British Museum, ni même avec des œuvres contemporaines de sa collection d'art contemporain du Moyen-Orient.Voir Time machine. Ancient Egypt and Contemporary Art, catalogue d'exposition (décembre 1994 - février 1995), Londres, The artists, and The Trustees of the British Museum, 1994.

4 GOB André, DROUGUET Noémie, La muséologie: histoire, développements, enjeux actuels, Paris, Armand Colin, 2003, 352 p.

5 "Les pratiques récentes de mixité entre art actuel et art ancien : le contemporain dans les musées ", PERSPECTIVE la revue de l'INHA, n 4, 2009, p. 503.
6 L'art contemporain du Moyen-Orient est une nouvelle catégorie de l'art contemporain apparue ces 10 dernières années. Cette catégorie est d'abord apparue dans le champ du marché de l'art au moment de la création des premières ventes aux enchères organisées à Dubaï par Christie's et Bonhams. Son usage s'est par la suite rapidement répandu dans le champ de la critique d'art et des musées. Nous renvoyons ici le lecteur à la première session intitulée "Defining the Middle East " du Symposium organisé en 2009 à la Tate à Londres et qui permet de saisir le caractère artificiel de cette nouvelle catégorie. Nous avançons que cette dernière doit essentiellement son succès ces dernières années à ce qu'elle permet d'éviter, se substituant à l'usage des termes « arabe " et " monde islamique ". Voir <http://www.tate.org.uk/about/projects/ contemporary-art-middle-east $>$.

7 La catégorie art islamique est une catégorie créée par les orientalistes européens à la fin du $19^{\mathrm{e}}$ siècle afin de désigner les objets, religieux ou séculiers, ayant été créés à partir du $7^{\mathrm{e}}$ siècle sous un patronage musulman par des artistes musulmans ou non musulmans. 
que comme des documents historiques et nourrissait les visions les plus essentialistes ${ }^{8}$. Dans cet article, nous souhaitons montrer que les expositions temporaires organisées par le British Museum et mettant en scène des œuvres d'art contemporain du Moyen-Orient perpétuent ces lectures ne privilégiant que les continuités au détriment des faits historiques de rupture et semblant ne chercher qu'à exposer la durée.

Le British Museum doit l'existence de sa collection d'art islamique historique à Augustus Wollaston Franks, conservateur au British Museum de 1851 à 1896. À l'époque de Franks, ces objets/œuvres sont des "documents d'art " puisqu'ils permettent aussi d'introduire des éléments d'histoire et d'anthropologie ${ }^{9}$. En 1855 , Franks précise que ces œuvres devaient n'être "pas uniquement de bonne qualité mais devaient porter une date, le nom d'un artiste ou une association historique intéressante... des documents sur les nombreuses branches d'art auxquelles elles appartiennent ${ }^{10}$ ".

Cette lecture historique et ethnographique de ces œuvres, au détriment de toute considération esthétique et artistique, perdure et concerne aussi les œuvres contemporaines. L'ancienne conservatrice de l'art islamique au British Museum (de 1983 à 2000), Rachel Ward, remarque en 2000 la persistance de l'influence de Franks, particulièrement sur la collection d'objets provenant du " monde islamique ».

8 Voir ABDALLAH Monia, «Exposer l'art contemporain du Moyen-Orient. Le British Museum face à ses collections ", Revue Intermédialités, Histoire et Théorie des Arts, des Lettres et des Techniques, $\mathrm{n}^{\circ} 15$ " Exposer/Displaying ", printemps 2010 ainsi que " Polysynthèse d'une caractérisation artistique entre 'Objets d'art' et 'Objets de civilisation' ", dans Cannibalismes disciplinaires. Quand l'histoire de l'art et l'anthropologie se rencontrent, (DUFRÊNE Thierry Dufrêne, TAYLOR Anne-Christine dir.), Actes du colloque Histoire de l'Art et Anthropologie organisé par l'INHA et le musée du quai Branly (21-23 juin 2007). Paris, éd. du Musée du quai Branly/INHA, 2009.

9 En ce sens, l'usage " conventionnel " du terme " islamique " pour lequel opte Oleg Grabar conviendrait certainement à Franks, Oleg Grabar explique en effet: " The adjective 'Islamic' should not be taken seriously in its literal sense. It is a conventional term to cover a broadly defined cultural entity over many centuries and the faith of Islam is only one aspect of that
Ward parle d'" effet durable que Franks a eu sur la présentation des œuvres provenant du Moyen-Orient au British Museum ${ }^{11}$ ". Les expositions temporaires associant art contemporain du Moyen-Orient et art islamique historique organisées au British Museum dans la galerie John Addis ou dans d'autres salles le démontrent.

\section{Les expositions temporaires organisées dans la galerie John Addis ${ }^{12}$}

Il existe au centre de la galerie John Addis un espace initialement conçu pour permettre au musée de montrer sa collection de miniatures. Cet espace sert aujourd'hui à l'organisation d'expositions temporaires qui juxtaposent souvent des œuvres appartenant aux collections du musée et d'autres empruntées à l'occasion de ces expositions. De 2004 à 2010, quatre expositions ont eu lieu et les uniques traces qu'elles laissent sont de brefs fascicules et quelques éléments sur le site Internet du musée.

La première, à l'automne 2004, faisait partie de l'exposition Soudan: Past and Present. Une œuvre calligraphique de l'artiste soudanais Osman Waqialla était exposée ainsi que des céramiques de l'artiste Mohammed Ahmed Abdallah Abbaro. D'autres œuvres calligraphiques de Hassan Musa faisaient partie de l'exposition Soudan: Past and Present, mais étaient exposées dans d'autres salles.

entity. ". Voir GRABAR Oleg, "The Aesthetics of Islamic Art ", (first published in 1993), Islamic Art and Beyond. Constructing the Study of Islamic Art, volume iii, 2006, p. 335.

10 Franks cité par Rachel Ward, "Augustus Wollaston Franks and the Display of Islamic art at the British Museum ", op. cit., p. 111. Je souligne.

11 WARD Rachel, "Augustus Wollaston Franks and the Display of Islamic Art at the British Museum ", op. cit., p. 105. 12 Voici la présentation de la galerie John Addis que l'on peut lire aujourd'hui sur le site Internet du musée: « La galerie John Addis - 7ème siècle avant J.C.- aujourd'hui: le terme 'islamique' est utilisé dans la salle numéro 34 afin de définir la culture de peuples vivant sur des terres dont la religion dominante est l'islam. L'exposition explore la foi, l'art, la calligraphie et la science musulmane ainsi que la prééminence de l'Islam parmi les cultures du monde ". <http://www. britishmuseum.org/explore/galleries/middle_east/room_34_ the_islamic_world.aspx>. 
La deuxième exposition temporaire présentée dans la galerie John Addis après 2004 s'intitule Making of The Master. The Art of Arabic Calligraphy. Organisée du 25 avril au 4 septembre 2005, son objectif était d'expliquer, par des exemples, les différentes étapes de l'enseignement de la calligraphie aujourd'hui.

Les exemples choisis étaient des exercices actuels effectués par des calligraphes d'aujourd'hui comme le calligraphe jordanien Nassar Mansour et leurs ijaza, c'est-à-dire des textes constitués de deux parties rassemblées sur une même carte décorée. La première partie calligraphiée par l'élève n'est pas signée, le texte est choisi avec le maitre calligraphe et il peut s'agir de versets du Coran ou de passages de la tradition du prophète (Hadith) ou de la description physique du prophète (Hilya). La seconde partie du texte contient divers éléments dont la phrase qui permettra à l'élève de signer de son nom "ajaztu bi wad' al-kataba ; cette phrase sera suivie de sa signature et du nom de son maître calligraphe ainsi que de la date. Parmi les exemples exposés figurent la Ijaza de Kouichi Fou'ad Honda, un calligraphe japonais converti à l'islam, la Ijaza d'une calligraphe turque Hilal Kazan ou encore celle d'un calligraphe algérien Muhammad al-Buhairi. Femme/homme, Maghreb/Mashreq de l'Islam étaient représentés venant ainsi illustrer l'idée d'" unité dans la diversité ». Les termes "still » et " today" étaient fréquemment utilisés, car le but était de montrer la continuité de cette tradition artistique: "Making of the Master célèbre cette tradition vivante en exposant la ijaza du maître calligraphe turc renommé, Hasan Celebi, basé à Istanbul ${ }^{13}$ ". Le British Museum renvoie dans son fascicule au site Internet de l'IRCICA ${ }^{14}$.

La troisième exposition temporaire s'intitule Iraq's Past Speaks to the Present. Organisée du 10 novembre 2008 au 15 mars 2009, elle présente côte à côte des artefacts archéologiques et des œuvres contemporaines provenant de collections privées ainsi que d'autres appartenant à la collection d'art contemporain " du Moyen-Orient » du musée. Cette exposition complète celle, plus importante, organisée par le British Museum dans d'autres salles et intitulée Babylon: Myth and Reality (13 novembre 2008 - 15 mars 2009). Pour cet espace de la galerie d'art islamique John Addis, les œuvres de 10 artistes irakiens et d'un artiste syrien sont rassemblées afin de montrer l'influence du « riche héritage archéologique et artistique de l'ancien Irak " connu par les anciens grecs comme la Mésopotamie. Ces œuvres se trouvent présentées aux côtés d'artefacts archéologiques de la région. L'œuvre Tower of Babel de Walid Siti, artiste kurde qui vit en Grande-Bretagne, se trouve ainsi présentée aux côtés d'un masque du démon Humbaba, le gardien de la forêt des cèdres dans la mythologie mésopotamienne, provenant de Sippar en Irak et daté de 18001600 av. J.-C., ainsi qu'aux côtés d'un pan de mur daté de 2500 av. J.-C. Dans la même vitrine, juste au-dessous, se trouve l'œuvre de l'artiste irakien Dia Al-Azzawi, The Mask of Gilgamesh. Cette œuvre s'inspire des récits épiques de Gilgamesh, roi d'Uruk ancienne ville de Mésopotamie. Tout en haut de cette même vitrine se trouve l'œuvre de l'artiste irakien Nasir Mounes, The Sumerian Love Chants. Cette œuvre fait partie d'une collection de 30 travaux qui s'inspirent d'un mythe sumérien autour de la fertilité et dont le sujet est le mariage de Dumuzi et Inanna.

Dans le fascicule qui accompagne cette exposition, on peut lire: " Ces artistes explorent des idées et traditions anciennes qui rappellent au spectateur non seulement la grande profondeur historique de leur pays mais aussi, comme le montre le travail de Issam Kourbaj, leur rappellent la destruction continuelle de notre héritage partagé à travers la guerre et la négligence $^{15}$ ".
13 Ibid., p. 2. Je souligne. (non paginé).

14 L'IRCICA : Islamic Research Center for Islamic History, Culture and Art a été fondé par l'Organisation de la Conférence islamique en 1979 et inauguré en 1980 à Istanbul. Voir <https://www.ircica.org/>.
15 Irad's Past speaks to the Present, John Addis Islamic Gallery, 10 novembre 2008 -15 mars 2009. Londres, The British Museum, 2008, p. 1 (non paginé). 
Alors qu'il ne s'agit aucunement d'art islamique dans le cadre de cette exposition temporaire ni d'aucune forme de ses traditions artistiques, cette exposition a pourtant bien lieu dans ces salles consacrées à l'Islam ${ }^{16}$. Ainsi, cette modernité artistique une fois associée par la géographie ou par la culture à l'Islam se trouve nécessairement présentée comme étant engagée dans un mouvement que l'historien de l'art Finbarr Barry Flood qualifie de " a paradoxical 'backto-the-future' model of Islamic modernity ${ }^{17}$ ". Flood cite le directeur du British Museum:

Le nouveau gouvernement intérimaire irakien devra considérer comment il définit l'identité irakienne. Et sans surprise, comme tous les autres gouvernements du Moyen-Orient, il se tournera vers des précédents historiques afin de définir le futur qu'il souhaite. Nul meilleur endroit que le British Museum pour passer en revue ces précédents ${ }^{18}$.

La quatrième exposition temporaire s'intitule
Zeeshan and Khadim Ali (23 mars - 18 octobre 2009) et fait partie d'un projet intitulé Innovation Through Tradition: Contemporary Iran and Pakistan. Ce projet inclut l'exposition Contemporary Iranian art: Saqqakhaneh and its legacy constituée d'une unique ouvre celle de l'artiste iranien Parviz Tanavoli (né en 1937) intitulée Heech exposée au milieu de la Grande cour du British Museum - et l'exposition Khosrow Hassanzadeh. Takhti: a modern Iranian Hero présentée dans la salle 3 à l'entrée du Musée.

16 Rappelons qu'en langue française, Islam avec un "I" majuscule désigne la civilisation islamique, alors que islam avec un "i" minuscule désigne la religion musulmane. 17 FLOOD Finbarr Barry, «From the Prophet to postmodernism? New World Orders and the end of Islamic art ", dans Making Art History: A Changing Discipline and its Institutions, (MANSFIELD Elizabeth ed.), Routledge. 2007, p. 40. 18 MACGREGOR Neil, "In the Shadow of Babylon ", The Guardian, juin 2004, p. 12, cité dans FLOOD Finbarr Barry, « From the prophet to postmodernism? New World Orders and the end of Islamic art ", op. cit., p. 40.
Comme son nom l'indique, cette quatrième exposition Safavids revisited: Sadegh Tirafkan (né en 1965), Muhammad Zeeshan (né en 1980) and Khadim Ali (né en 1978) tente de montrer la continuité de la tradition de la miniature en Iran ainsi qu'au Pakistan:

Elle [cette exposition] met en relief le pouvoir imprescriptible des arts des safavides et des miniatures persanes et montre comment les artistes créent des œuvres inspirées par les styles et les thèmes qu'elles expriment, leur donnant une nouvelle résonance dans le monde d'aujourd'huii ${ }^{19}$.

Ainsi, on peut lire dans le texte intitulé Reinventing miniature écrit par Hammad Nasar et publié au sein du fascicule qui accompagnait cette exposition:

Ce qui rend le phénomène de résurgence de la miniature contemporaine intéressant n'est pas son acceptation au sein du panthéon de la pratique de l'art contemporain mais plutôt son principe fondateur qui va à l'encontre de la conception euro-américaine de l'avant-garde. Les praticiens n'innovent pas en rejetant ce qui s'est passé avant eux mais à travers la maîtrise d'habiletés traditionnelles qu'ils dépassent ensuite $^{20}$.

$\mathrm{La}$ " miniature " et son rapport à la tradition de la miniature moghole est probablement pour les artistes pakistanais tels que Zahoor ul Akhlaq (1941-1999) et Imran Qureshi (1972) ce qu'est l'usage de la lettre arabe et son rapport à la tradition de la calligraphie islamique pour les artistes arabes, iraniens et turcs: une avant-garde. Une avant-garde qui, paradoxa-

19 Innovation Through Tradition: Contemporary art from Iran and Pakistan. Londres, The British Museum et the Altajir Trust, 2009, p. 10. Je souligne.

20 NASAR Hammad, "Reinventing miniature ", dans Innovation Through Tradition: Contemporary art from Iran and Pakistan. Londres, The British Museum et the Altajir Trust, 2009, p.12. 
lement, cherchait dans les années 1960 et 1970 un moyen de rompre avec ce qui était perçu et vécu comme une rupture, celle suscitée par la création des écoles des beaux-arts au moment de la colonisation et l'introduction de la " modernité artistique occidentale ${ }^{21}$ ". Cet usage de la lettre arabe et de l'esthétique formelle de la miniature dans la modernité artistique des pays d'Islam relève moins d'un esprit de préservation et d'un retour à la tradition, que d'un esprit de rupture et d'innovation. Or, ce qui semble évident aujourd'hui, c'est que la « miniature " comme la " calligraphie " constituent des arguments iconographiques pour les discours - non homogènes - qui tentent d'établir, ou de trouver, une continuité artistique entre cet art moderne et contemporain du Moyen-Orient et l'art islamique historique, un art condamné à n’avancer que vers le passé.

\section{Retour historique sur l'introduction de la lettre arabe dans l'art moderne et contemporain du Moyen-Orient}

\section{En 1952, l'artiste iraquienne Madiha Omar} inaugure formellement à Bagdad l'usage de la lettre arabe dans une production artistique moderne. Cette artiste fut encouragée dès 1949 dans cette recherche formelle par l'historien de l'art islamique Richard Ettinghausen qui organise, cette même année 1949, une exposition de ses œuvres à la Georgetown Public Library de Washington. Madiha Omar est la première artiste à rédiger une déclaration qui revendique les possibilités plastiques de l'alphabet arabe. Ce manifeste s'intitule Arabic Calligraphy: An Element of Inspiration in Abstract Art. Comme le titre l'indique, la production artistique qui en découle est considérée par l'artiste comme relevant de l'art abstrait. La référence à la calligraphie arabe n'implique pas une volonté de retour à cette tradition artistique propre à l'art islamique historique.

De même lorsqu'en 1970 l'artiste iraquien Shakir Hassan Al Saïd rédige son manifeste à Bagdad sous le titre Albu'd el wahid (L'Unique dimension), il marque l'apparition d'un mouvement en double rupture ${ }^{22}$. D'abord avec ce qui est communément admis comme introduisant la modernité artistique "en terres d'Islam ", c'est-à-dire la création, au moment de la colonisation, des différentes écoles des beaux-arts et l'enseignement qui y est offert (1908: création d'une école des beaux-arts au Caire; 1937 au Liban; 1941 en Iraq et 1959 en Syrie) $)^{23}$. Cette première rupture s'est accompagnée, dans le cadre de ce mouvement né en Iraq et auquel adhèrent de nombreux artistes arabes ${ }^{24}$, de la volonté de reconquérir un passé qui serait proprement arabe, c'est-à-dire préislamique, afin d'affirmer une identité arabe contemporaine dans un contexte général: celui du nationalisme arabe. Cette volonté se manifeste plastiquement par le développement d'une esthétique liée aux possibilités formelles qu'offre la lettre arabe. Elle n'a volontairement pas fait le choix de revenir à une identité islamique mais bien à une identité ethnique arabe préislamique. Le nom
21 Sur le retour à la tradition de la miniature moghole dans l'œuvre de certains artistes pakistanais, voir l'article de HASHMI Salima, "Spinning Stories. The Art of Pakistani Women Miniaturists ", A Thousand and One Days. Pakistani Women artists, exposition Honolulu Academy of Arts, 13 octobre-11 décembre 2005. Milan, Silvana Editoriale, 2005, p. 23-31. (Je remercie Ana Cecilia Hornedo Marin pour m'avoir indiquée ce catalogue). Ce retour à la miniature ne concerne pas que les artistes pakistanais. L'artiste algérien Mohammed Racim (1896-1975), par exemple, s'est aussi inspiré de l'art de la miniature intégrant la perspective et le modelé dans la miniature persane. Voir Mohammed Racim: miniaturiste algérien, exposition organisée par l'Institut du Monde Arabe et le Musée national des Beaux-arts d'Alger (3-29 mars 1992). Paris, I.M.A., 1992, 46 p.

22 Manifeste signé notamment par Dia Al-Azzawi et Rafa Al-Nasiri.
23 Si l'on se réfère à la distinction que fait Shigemi Inaga du choix devant lequel se sont retrouvés les artistes non occidentaux qui souhaitaient établir un art national - entre rester " fidèles à la forme et infidèles à l'esprit d'avant-garde ou, inversement, fidèles à l'esprit et infidèles à la forme " -, il semble que le modernisme artistique dans ces pays arabes se soit montré fidèle à " l'esprit moderne " en se positionnant ainsi en rupture avec cette " modernité importée " du début du $20^{\text {e }}$ siècle. Voir Shigemi Inaga, "L'impossible avant-garde au Japon ", dans Transcultura, Connaissance et réciprocité, Louvain, Presses universitaires de Louvain, 1988, p. 199. 24 Dans son article, Buland Al-Haidari affirme que des artistes soudanais avaient commencé à travailler la lettre arabe dès le début des années 1950. Voir AL-HAIDARI Buland, "Calligraphy in Modern Arab Art ", dans Arts \& The Islamic World, vol. 1, n 1, Londres, hiver 1982/1983, p. 22. 
donné à ce mouvement est celui de Hurufiyya (lettrisme), un nom formé à partir du mot Harf qui signifie " lettre " en arabe : il a délibérément été choisi afin de distinguer cet usage contemporain de l'alphabet arabe de son usage dans la tradition de la calligraphie islamique. Dans ce sens, ces artistes se sont ainsi désignés comme des hurufiyyoun ce que l'on pourrait traduire par " des travailleurs de la lettre", des "lettristes " et non plus comme des Khattatoun - mot arabe pour désigner les calligraphes.

Ce mouvement a donc pour corollaire une seconde rupture, du moins implicite: il ne s'agit pas de construire une identité fondée sur une tradition artistique islamique mais bien de participer, par l'art, à la construction d'une identité nationale arabe contemporaine. Précisons que lors de l'exposition L'unique dimension organisée à Bagdad en 1971, dont l'affiche fut conçue par Dia Al-Azzawi, figuraient aux côtés des œuvres contemporaines de ces artistes des exemples d'œuvres de "nature historique et islamique ». Dia Al-Azzawi en donne cette explication : " afin de donner forme à la vision actuelle des caractères arabes en tant qu'éléments esthétiques à grand potentiel ${ }^{25}$ ". Par conséquent, la présence de ces œuvres historiques se fonde sur le potentiel artistique de la lettre arabe et non pas sur l'inspiration esthétique de la calligraphie islamique.

Dans ce même contexte, un mouvement similaire a vu le jour en Iran au début des années 1960 sous le nom de Saqqakhaneh. Ce mot signifie en persan "fontaine d'eau " et a été forgé en 1962 par le journaliste Karim Emami. Les œuvres des artistes qui formaient cette école Saqqakhaneh intégraient les symboles populaires de la culture shiite, symboles religieux et folkloriques. Cette période reste considérée comme fondamentale pour l'art iranien au $20^{\mathrm{e}}$ siècle. Parviz Tanavoli et Hossein Zenderoudi sont les deux principaux artistes de ce mouvement qualifié de " pop art spirituel » du fait de l'introduction d'éléments de la culture populaire dans l'art. Kameran Diba, premier directeur du Musée d'art contemporain de Téhéran, écrit à ce propos dans le catalogue Contemporary Art from the Islamic World: « Il y a un parallèle entre SaqqaKhaneh et le Pop Art, si nous simplifions, le Pop Art est un mouvement qui considère les symboles et les outils de la société de consommation de masse comme une force culturelle pertinente et qui a de l'influence. Les artistes de SaqqaKhaneh ont examiné les croyances intérieures et les symboles populaires issus de la religion et de la culture de l'Iran, et qui étaient probablement consommés de la même manière que les produits industriels en Occident (mais pour des raisons différentes et dans des circonstances distinctes) $)^{26} "$

En Turquie, certains artistes comme Erol Akyavas retournent à l'écriture arabe. Pour ces artistes de pays non arabes (Iran et Turquie), il s'agissait d'affirmer une identité en réaction à la colonisation et à ce qu'elle avait importénotamment l'alphabet latin (dès 1928, en Turquie, sous Kemal Atatürk). Ce fut donc pour des raisons historiques et culturelles que des artistes, qu'ils soient de pays arabes ou non, se sont tournés vers la lettre arabe. Si les artistes des pays arabes cherchaient, par l'usage de cette lettre, à affirmer une identité arabe, les artistes iraniens et turcs cherchaient, par contre, une identité nationale qui leur fut propre, en rupture avec la colonisation.

\section{Le British Museum et son interprétation de l'usage actuel de la lettre arabe comme continuité de la calligraphie islamique: considérations à partir de deux expositions temporaires organisées par le British Museum}

25 AL-AZZAWI Dia, «Arabic Characters as an Artistic Form ", The Influence of Calligraphy on Contemporary Arab Art [Londres, The Iraqi Cultural Centre, 20 février-14 mars 1980]. Londres: The Iraqi Cultural Centre, non paginé.
26 DIBA Kameran, «Iran », dans Contemporary Art from the Islamic World, ed. par W. ALI, Londres, Barbican Centre, Septembre-Octobre 1989. Amman: The Royal Society of Fine Arts, 1989, p. 153. 


\section{Première exposition: Mightier than the Sword. Arabic script: beauty and meaning}

Le site Internet du British Museum propose, sous la rubrique World Cultures puis Islamic Middle East et The Islamic World, la visite virtuelle d'une partie de ses collections. L'une des sections est intitulée Arabic Script: Mightier than the Sword - un titre qui renvoie à ce précepte de la calligraphie: The pen is mightier than the sword qui lui-même exprimerait " la reconnaissance universelle du pouvoir du mot écrit ". Cette visite procède d'une approche des objets à la fois diachronique - puisqu'elle présente de nombreux exemples de calligraphie islamique sur céramiques, textiles, parchemin et autres supports depuis le Moyen Âge jusqu'à nos jours et synchronique, puisque le travail d'artistes actuels, tout aussi bien malais, irakiens que tunisiens, est introduit en ces termes dans la section Contemporary art: « Les formes de la lettre arabe continuent d'inspirer les artistes aujourd'hui. Certains artistes contemporains sont des calligraphes formés à la pure tradition. Mais d'autres se sont mis à utiliser l'écriture arabe ou des imitations d'écriture de façon extrêmement inventive ». Une telle présentation laisse supposer que les artistes d'aujourd'hui, quelle que soit la nature de leur usage de la lettre arabe, poursuivraient la tradition de la calligraphie islamique.

Cette exposition est la version virtuelle d'une exposition itinérante organisée par le British Museum avec l'aide de Altajir World of Islam Trust sous le titre Mightier than the Sword. Arabic script: beauty and meaning. Le catalogue publié à l'occasion de sa tenue en Australie au Ian Potter Museum of Art de l'Université de Melbourne du 22 mars au 25 mai 2003 se présente comme suit: six premières sections dans lesquelles ne figurent aucune ouvre contemporaine: I. Islam and the word of God; II. Beauty and diversity; III. Contact with Europe: Arabic script as pattern; IV. Islam in China and
Indonesia; v. The art of the calligrapher; vI. Virtuosi scripts on paper. Elles sont suivies d'une septième section intitulée The contemporary art of writing and calligraphy dans laquelle figurent huit œuvres contemporaines réalisées par les sept artistes suivants: Ali Omar Ermes, Nja Mahdaoui, Ahmed Moustafa, Ghani Alani, Nassar Mansour, Khaled Ben Slimane et Leila Shawa. Deux autres œuvres contemporaines créées par Osman Waqialla et Rachid Koraichi sont présentées dans la dernière section intitulée The Power of the word aux côtés d'objets datés du $\mathrm{XI}^{\mathrm{e}}$ au $\mathrm{XX}^{\mathrm{e}}$ siècle et qui sont considérés comme ayant des pouvoirs magiques telles que des amulettes des XVIII ${ }^{\mathrm{e}}$ et XIX ${ }^{\mathrm{e}}$ siècles. Venetia Porter écrit au sujet de ces deux dernières sections: "L'art contemporain de l'écriture et de la calligraphie inclut des exemples marquants de nombreux artistes moyen-orientaux. La section finale 'Le pouvoir du mot' aborde le côté populaire de l'islam à travers une variété d'objets incluant des bols magiques et des amulettes populaires imprégnés de pouvoirs protecteurs $^{27}$ ".

Le fil conducteur de cet éclectisme est l'écriture arabe perçue comme " arabe-islamique ". En effet, comme l'explique Neil MacGregor, directeur du British Museum à l'époque, le lien entre l'écriture arabe et l'islam est inextricable: "Cette exposition célèbre la beauté du mot, du mot arabe sanctifié par l'islam et adopté ensuite de l'Afrique de l'Ouest jusqu'à l'Indonésie ". Toutes ces œuvres anciennes et contemporaines " offrent un aperçu de l'une des grandes réalisations de la civilisation islamique ${ }^{28}$ ". Or, si ces œuvres contemporaines font partie "des réussites de la civilisation islamique ", cela implique que la " civilisation islamique " perdure aujourd'hui. (Cette exposition sera par la suite présentée au Musée des Arts islamiques de Malaisie à Kuala Lumpur au printemps 2004).

28 MACGREGOR Neil, "Preface ", Mightier than the sword. Arabic script: beauty and meaning, op. cit., p. 4.
27 PORTER Venetia, "Introduction ", Mightier than the sword. Arabic script: beauty and meaning, catalogue d'exposition, The Ian Potter Museum of Art, the university of Melbourne, 22 mars-25 mai 2003. Victoria, The Ian Potter Museum of Art, the University of Melbourne, mars 2003, p. 8 . 


\section{Deuxième exposition: Word into Art. Artists of the Modern Middle East}

Du 18 mai au 2 septembre 2006, le British Museum présente dans la salle 35 au-dessus de la Grande cour, l'exposition Word into Art. Artists of the Modern Middle East qui sera par la suite itinérante ${ }^{29}$. Cette exposition fait partie du programme mis en place par le musée pour la saison 2006 et qui est intitulé Middle East Now. Son objectif est de faire connaître le "Moyen-Orient $d$ 'aujourd'hui " à travers son histoire et ses cultures. Des événements musicaux, cinématographiques ainsi que des documentaires historiques et politiques comme le dernier entretien accordé par Edward Saïd accompagnent ce programme. Cinq événements concernent les arts visuels :

- une journée d'études intitulée Middle East Art Now qui réunit aux côtés de chercheurs les artistes suivants: Farhad Moshiri, Charlie Rabbah et Kamal Boullata, artistes qui utilisent la lettre arabe dans la plupart de leurs œuvres;

- deux conférences de l'artiste Jila Peacock sur l'histoire de l'art de la calligraphie dans le monde islamique et plus particulièrement persan;

- une soirée Middle East Now: words, images, music avec l'artiste et poète Etel Adnan ainsi que les artistes Said Farhan et Mai Ghoussoub;

- enfin, une démonstration de calligraphie menée par quelques artistes de l'exposition Word into Art.

Ces cinq événements liés aux arts visuels se rapportent nécessairement à l'usage de la lettre arabe par les artistes contemporains choisis pour ces événements - artistes qui seraient représentatifs des artistes " du Moyen-Orient d'aujourd'hui ». Cet usage de la lettre arabe est associé à l'art de la calligraphie islamique ce que confirme par ailleurs l'organisation thématique de l'exposition principale Word into Art: ainsi le catalogue reprend l'organisation spatiale de l'exposition en quatre salles thématiques: 1. A Sacred Script; 2. Literature and Art; 3. Deconstructing the Word; 4. Identity, History and Politics. C'est donc à travers le mot arabe dont la filiation commencerait avec l'islam que s'organise l'exposition. Venetia Porter, conservatrice au British Museum, explique que cette écriture est " non seulement un moyen de communication - dans une culture où la représentation figurative n'était pas encouragée - mais elle est aussi une source majeure de créativité et un trait distinctif de l'art des pays d'Islam ${ }^{30}$ ".

Les interprétations que donne le British Museum de cet usage actuel de la lettre arabe par des artistes contemporains viennent conforter des liens pour le moins problématiques. En effet, ces deux expositions Mighthier than the Sword. Arabic script: beauty and meaning et Word into Art. Artists of the Modern Middle East ainsi que l'introduction au sein de la galerie John Addis d'œuvres contemporaines usant de la lettre arabe souhaitent sans doute illustrer la préservation d'une continuité culturelle en " terres d'Islam " et, peut-être, rendre également hommage à une histoire spécifique du goût et de la création, mais de tels discours renforcent également l'idée selon laquelle il existerait une " famille spirituelle ", une continuité d'" esprit ", une " essence " et ne correspondent d'ailleurs qu'à la manière dont seuls certains artistes envisagent leur pratique contemporaine.

Le point central de cette interprétation, c'est-àdire de l'interprétation de l'usage de l'écriture arabe par des artistes contemporains comme étant la continuité de la tradition de la calligraphie dans la civilisation islamique passée ainsi que son association à la notion de "modernité " ("Artists of the Modern Middle East "), implique l'idée nouvelle que cette civilisation perdure. 


\section{Problèmes posés par cette interprétation ${ }^{31}$}

Selon Venetia Porter, la nostalgie expliquerait la volonté des artistes "du Moyen-Orient " de revenir à un art islamique " authentique ": "Il y a une volonté évidente de créer un pont avec le passé et c'est une préoccupation récurrente dans de nombreuses œuvres d'artistes du Moyen-Orient ", peut-on ainsi lire en introduction au catalogue Word into Art ${ }^{32}$. L'usage de la lettre arabe par ces artistes contemporains s'y trouve ensuite interprété comme " une prise de conscience témoignant d'une volonté de revenir à des thèmes islamiques ".

Pourtant, lorsqu'une critique d'art demande à l'artiste Rachid Koraichi - dont une œuvre était présentée à l'exposition Word into Artlors d'un entretien en 1993: "Dans votre usage de la calligraphie, y a-t-il un intérêt de vous mesurer à la tradition? ", la réponse de l'artiste est catégorique: «Mon travail n'est pas de la calligraphie ». Puis, il insiste sur le fait qu'une œuvre qui s'inspire de la calligraphie n'est pas une calligraphie ${ }^{33}$ et poursuit:

En regardant mes plus récentes pièces, vous pouvez voir que toutes les caractéristiques de l'écriture arabe ont été inversées, montrant que je n'ai aucun intérêt à ce qu'elles soient lues, dans ce sens, mon intention est à l'opposé de celle de la calligraphie. Ce que je souhaite est que le spectateur ressente les effets d'un morceau de texte uniquement d'un point de vue esthétique $^{34}$.
De même pour l'artiste irakien Dia Al-Azzawi dont les œuvres sont parmi les premières à avoir intégré la collection d'art contemporain " du Moyen-Orient " du British Museum, son œuvre ne peut être réduite à son usage de la lettre arabe :

Je crois en l'utilisation d'éléments visuels en peinture comme éléments primaires: l'écriture arabe devrait en faire partie. Je ne pense pas qu'une peinture devienne arabe à travers l'utilisation de l'écriture arabe. L'identité de la peinture provient d'un ensemble d'éléments, pas seulement de textes ou d'ornementations ${ }^{35}$.

Par conséquent, le premier problème que pose l'interprétation systématique de l'usage de la lettre arabe par des artistes contemporains comme étant une forme de continuité de la tradition de la calligraphie islamique est d'ordre esthétique. Il concerne précisément l'esthétique analytique. En effet, pour Dia Al-Azzawi tout comme pour Nelson Goodman dans son article "Quand y a-t-il art?", les symboles visuels « qu'ils soient là pour rehausser ou pour distraire sont extrinsèques à l'œuvre ellemême ". Al-Azzawi semble ici redire à sa manière ce qu'écrivait Goodman : "L'œuvre d'art est ce qu'elle est plutôt que ce qu'elle symbolise ". De là, comment attribuer une "identité "- nationale, ethnique, religieuse ou culturelle à une œuvre d'art?

Le deuxième problème que pose cette interprétation est d'ordre sociologique et anthropologique. En effet, une fois confrontée aux

34 Rachid Koraichi cité dans VEGA CORDERO Leticia, "Meeting with Rachid Koraichi ", Third Text, $\mathrm{n}^{\circ}$ 25, hiver 1993-1994, p. 62.

35 AL-AZZAWI Dia, propos cités dans "Introduction ", Word into Art. Artists from the Modern Middle East. Londres, The British Museum Press, 2006, p. 17.
31 Pour un article critique résumant les problèmes que pose l'exposition Word into Art. Artists of the Modern Middle East, voir Hassan Khan, "Word into Art: Artists of the Modern Middle East - The British Museum ", BIDOUN. ARTS AND CULTURE FROM THE MIDDLE EAST, vol. 1, $\mathrm{n}^{\circ} 8$, automne 2006, p. 114-115.

32 PORTER Venetia, "Introduction", Word into Art, op. cit., p. 18.

33 Rachid Koraichi explique: "Calligraphy has its specific rules and techniques. You can see in the pieces I have made, aside from the use of fragments of manuscrits, that there is a difference between calligraphy and the work of art that comes from calligraphy. ". Voir VEGA CORDERO Leticia Cordero, "Meeting with Rachid Koraichi ", Third Text, $\mathrm{n}^{\circ}$ 25, hiver 1993-1994, p. 62. 
contradictions des identités individuelles d'artistes comme l'artiste libyen Ali Omar Ermes, cette interprétation semble réductrice. Ainsi, tantôt Ali Omar Ermes revendique une identité " arabe " et " islamique » ainsi que l'existence d'un " art islamique contemporain " ${ }^{36}$, tantôt il dénonce le caractère réducteur de certaines taxinomies et écrit:

Il existe une conception de l'artiste qui serait originaire du monde musulman ou d'Orient qui enferme son travail dans un carcan comme si, en raison de ses origines, il ne pouvait contribuer au monde extérieur plus large; comme s'il ne pouvait contribuer qu'à son monde immédiat, c'est-à-dire le monde musulman ou une communauté musulmane particulière ${ }^{37}$.

Pour James W Allan, il serait erroné de considérer Ali Omar Ermes comme un calligraphe traditionnel. Ermes se considère lui-même comme un artiste peintre et affirme: "I am not a calligrapher - I am an artist. ${ }^{38}$ ". Selon Allan, Ermes une plus grande liberté que celle qui est permise à un calligraphe ${ }^{39}$ ". De même, l'artiste refuse d'introduire dans ses œuvres des versets du Coran. Dale Egee en parle de la façon suivante:

L'autre position intransigeante [d'Ali Omar Ermes] se situe au niveau du thème calligraphique. Je lui ai demandé de peindre une sourate du Coran pour un client. Il a refusé expliquant qu'il ne reproduisait pas de sourates du Coran

36 Le 4 juillet 1999, dans le cadre de la Semaine culturelle islamique, Ali Omar Ermes présente au Centre Culturel d'Irlande une conférence intitulée: "Contemporary Islamic Art ". Pourtant, Venetia Porter, conservatrice au British Museum, écrit au sujet d'Ali Omar Ermes: "Ermes sees himself rightly as much a part of an international art movement as an Arab or an 'Islamic' painter, although these elements are obviously present in his work and he is himself a committed Muslim ". Voir Venetia Porter, "A lifetime of Painting ", Ali Omar Ermes In Context. Londres, Art Advisory Associates, 2003, p. 15.

37 ERMES Ali Omar, "Interview by Sajid Rizvi: Art and Ideas ", Ali Omar Ermes In Context. Op. cit., p. 30.

38 DALE Egee, "Ali Omar Ermes ", Ali Omar Ermes In Context, op.cit., p. 14.

ne sachant pas où celles-ci seraient exposées, ni même qui pourrait les côtoyer ou les profaner ${ }^{40}$.

Or, il arrive pourtant à Ali Omar Ermes d'apposer avant sa signature cette formule traditionnelle hadha min 'amal (ceci est le travail de) qui était utilisée dans les siècles passés par les calligraphes qui signaient leurs œuvres. Dans cette confrontation entre art contemporain et art ancien, Jean-Hubert Martin rappelait dans un entretien effectué en 2008 l'importance qu'il y a de s'intéresser à la complexité du processus créatif, de même qu'à la richesse paradoxale de toute individualité artistique, et disait à ce sujet: « Il faut une approche qui soit beaucoup plus proche de la création, plus ouverte à tout ce qu'il y a de chaotique, d'anachronique, d'irrationnel dans celle-ci ${ }^{41}$ ".

Par ailleurs, en 1996, le British Museum expose au sein de la galerie John Addis d'art islamique une œuvre de l'artiste iranienne Shirazeh Houshiary où l'écriture arabe devient peu à peu indéchiffrable. Cette œuvre s'inspire du mysticisme soufi du poète Jalal al-Din Rumi. La réaction négative de l'artiste quant à cet emplacement est significative. Frances Carey raconte:

Ceci causa un grand trouble à Houshiary en raison de son aversion pour l'appellation qu'on lui porte "d'artiste islamique"; il était clair qu'elle aurait largement préféré être considérée dans un contexte plus neutre comme celui de la galerie des estampes et des dessins qui est géné-

39 ALLAN James W, "Introduction ", Ali Omar Ermes - Art and Ideas, op. cit., p. 7. De même, Mary Patrick écrit à propos du travail d'Ali Omar Ermes: "But for the calligraphic overlay the effect is evocative of contemporary Works of modern art ". Voir PATRICK Mary, "Ali Omar Ermes - A Fusion of Unusual Energy ", Eastern Art Report, 16-31 mars 1989, p. 15. 40 DALE Egee, "Ali Omar Ermes ", Ali Omar Ermes In Context, op. cit., p. 14.

41 ISUANI Yanina, op.cit., p. 113. 
ralement dédiée aux œuvres d'artistes aux origines culturelles européennes ${ }^{42}$.

Par conséquent, la galerie John Addis n'est pas perçue, au moins par les artistes eux-mêmes, comme un espace d'exposition neutre. Cet espace d'exposition expose à son tour l'artiste à une labellisation qui le situe dans une entité culturelle et religieuse " autre " et l'exclut simultanément d'un espace d'expression commun considéré plus proche d'une forme de neutralité. La présence de cette œuvre d'Houshiary au sein de la galerie John Addis se fonde, tout comme l'exposition Word into Art, sur l'interprétation de l'usage de la lettre arabe par des artistes contemporains comme étant une forme de continuité de la tradition de la calligraphie islamique. Or, pour John Carswell, cet usage lisible ou illisible de la lettre arabe serait « factice " et aurait très peu de choses en commun avec la calligraphie islamique. C'est ce qu'il explique en 1989 à la suite de cette question: "Q. Plusieurs artistes actifs sur la scène islamique internationale ont passé leurs vies à l'étranger. Ils sont souvent critiqués pour des productions souvent étrangères à leurs propres cultures. » La réponse de Carswell est catégorique: «Absolument. Et il y a autre chose qui me hérisse les poils : c'est l'art moderne qui est basé sur la calligraphie islamique car je pense que c'est une imposture. Il s'agit de l'utilisation de l'écriture et de son renversement pour en faire des images ${ }^{43}$ ".

On le voit, cette interprétation du British Museum ne fait pas l'unanimité. Elle crée une " attitude à l'égard de ces images " comme "les sons d'un diapason qui définiraient la tonalité de chaque image, le monde pictural dans lequel elle doit être comprise " pour reprendre les termes de l'historien de l'art islamique Oleg Grabar sur un tout autre sujet ${ }^{44}$. Il est paradoxal qu'un artiste tel que Dia Al-Azzawi qui a signé en octobre 1969 à Bagdad le Manifeste Vers Une Nouvelle Vision - dans lequel on pouvait lire: «La révolution qui avance et qui produit le modèle révolutionnaire est aussi l'art du futur, un art qui étonne et réalise la vision $\left(r u^{\prime} y a\right)$ nouvelle ${ }^{45}$ »- voit aujourd'hui son art constamment ramené à du " déjà vu ". Ces œuvres contemporaines n'étant expliquées que par rapport à ce qui les précède.

Malgré les problèmes que pose cette interprétation, elle témoigne de façon remarquable du rôle primordial que joue la "calligraphie " dans l'idée de permanence de l'art islamique. La BBC ne présentait-elle pas l'exposition Word into Art comme " a good introduction to new Islamic art ${ }^{46}$ " ? Ainsi, cette idée d'un " nouvel art islamique " se fonde en grande partie sur l'interprétation de l'usage plastique de la lettre arabe comme une forme de continuité de la calligraphie islamique. La " calligraphie » devient donc une condition de l'existence d'un art islamique aujourd'hui et cette interprétation du British Museum ainsi que ce choix muséographique d'associer de façon systématique la présentation d'art contemporain du Moyen-Orient avec des œuvres de l'art islamique historique se répandent dans la majorité des grands musées d'Europe et des États-Unis oubliant que dans la création " rien n'est linéaire, mais plutôt chaotique et inattendu ${ }^{47}$ ».
42 CAREY Frances, "Elective Affinities. Collecting Contemporary Culture in the British Museum ". Conférence présentée à Collecting Now: A Conference in Collecting the Modern, British Museum and University of Kingston, March 2003, <http.www.kingston.ac.uk/-kul5905/BM/PDFs/ Elective\%20Affinities>

43 "John Carswell - Man with Art on His Mind", op. cit., p. 13.

44 Oleg Grabar explique par ces phrases citées les " grands thèmes de la peinture persane ". Voir GRABAR Oleg, La peinture persane. Une introduction. Paris, Presses Universitaires de
France, coll. «ISLAMIQUES », 1999, chapitre IV, p. 97. 45 Voir la traduction de ce manifeste dans NAEF Silvia, À la recherche d'une modernité arabe. Genève, Ed. Slatkine, 1996, Annexe i: Manifestes artistiques, p. 375-380. 46 "But for anyone knowing little about the art scene in cities like Tehran or Cairo this is a good introduction to new Islamic art whatever its origins". Voir " The word in contemporary Islamic art ", BBC News : <http://news.bbc.co.uk/2/hi/ middle_east/4987284.stm>.

47 Jean-Hubert Martin dans ISUANI Yanina, Op.cit., p.113. 


\begin{abstract}
The confrontation between contemporary and ancient art, within the framework of temporary exhibitions or in the context of permanent collections, is not new, and examples are numerous. This article shows, through a description of a variety of temporary exhibitions organized by the British Museum, bringing together contemporary Middle Eastern and ancient Islamic art, the ideological consequences of such juxtapositions which consistently favour continuity over rift.
\end{abstract}

